\title{
The effects of cell death-inducing DNA fragmentation factor- $\alpha$-like effector $C$ (CIDEC) on milk lipid synthesis in mammary glands of dairy cows
}

\author{
Yang Yang, ${ }^{*}$ Ye Lin, ${ }^{*} †$ Xiaoyu Duan, ${ }^{*}$ He Lv, ${ }^{*}$ Weinan Xing, ${ }^{*}$ Qingzhang Li, ${ }^{*} \dagger$ Xuejun Gao, ${ }^{*} \dagger$ \\ and Xiaoming Hou*1 \\ ${ }^{*}$ College of Life Science, Northeast Agricultural University, Harbin 150030, China \\ tKey Laboratory of Dairy Science of Education Ministry, Northeast Agricultural University, Harbin 150030, China
}

\begin{abstract}
Adequate lipid synthesis by the mammary gland during lactation is essential for the survival of mammalian offspring. Cell death-inducing DNA fragmentation factor- $\alpha$-like effector C (CIDEC) is a lipid droplet-associated protein and functions to promote lipid accumulation and inhibit lipolysis in mice and human adipocytes. However, the function of CIDEC in regulation of milk lipid synthesis in dairy cow mammary gland remains largely unknown. In this study, 6 multiparous Holstein cows (parity $=3$ ) in early lactation were allocated to high-fat milk (milk yield 33.9 $\pm 2.1 \mathrm{~kg} / \mathrm{d}$, milk fat $>3.5 \%, \mathrm{n}=3$ ) and low-fat milk (milk yield $33.7 \pm 0.5 \mathrm{~kg} / \mathrm{d}$, milk fat $<3.5 \%, \mathrm{n}=3$ ) groups according to their milk fat content. Lactating cows were slaughtered at $90 \mathrm{~d}$ in milk and mammary tissues were collected to detect CIDEC localization. Immunofluorescence staining of sections of lactating mammary glands with high- and low-fat milk showed that CIDEC was expressed in the cytoplasm of epithelial cells and localized to lipid droplets. Lipid droplets and CIDEC protein were also detected in isolated lactating mammary epithelial cells of dairy cows. Immunostaining of CIDEC in isolated mammary epithelial cells also confirmed its presence in the nucleus. The knockdown of CIDEC in cultured bovine mammary epithelial cells decreased milk lipid content and reduced expression of genes associated with mammary de novo fatty acid synthesis, short- and long-chain intracellular fatty acid activation, triacylglycerol synthesis, and transcription regulation. These genes included those for acetyl-CoA carboxylase (ACC, $-60 \%$ ), fatty acid synthase (FASN, $-65 \%$ ), acyl-CoA synthetase short-chain family member 2 (ACSS2, -50\%), acyl-CoA synthetase long-chain family member 1 (ACSL1, -30\%), diacylglycerol acyl-
\end{abstract}

Received June 2, 2016.

Accepted December 19, 2016.

${ }^{1}$ Corresponding author: xiaominghou@126.com transferase 1 (DGAT1, -60\%), sterol regulatory element-binding protein 1 (SREBP1, $-45 \%$ ), and SREBP cleavage activating protein (SCAP, $-66 \%$ ). Conversely, in cells overexpressing $C I D E C$, triacylglycerol content was increased, and transcription of those genes involved in milk lipid synthesis was coordinately upregulated. These results suggest that CIDEC plays an important role in regulating milk lipid synthesis in dairy cow mammary gland via a mechanism involving gene expression, which provides further insight into the mechanisms regulating mammary lipogenesis in ruminants.

Key words: dairy cow, mammary gland, milk lipid, cell death-inducing DNA fragmentation factor- $\alpha$-like effector C

\section{INTRODUCTION}

Lipids, primarily triacylglycerols (TAG), are major milk constituents of most mammals, providing a large percentage of the calories, essential fatty acids, and bioactive lipids required for neonatal growth and development (McManaman, 2009). Lactating mammary gland synthesizes large amounts of TAG from fatty acids (FA) derived from the blood and from de novo lipogenesis (Emery, 1973). In cows, about half of the milk FA are derived from de novo synthesis (Bauman and Griinari, 2003).

Milk lipid synthesis is a complicated process in vivo and is highly regulated by gene expression. Cell deathinducing DNA fragmentation factor- $\alpha$-like effector C (CIDEC) is a lipid droplet-associated protein (Tan et al., 2014). It is highly expressed in both white and brown adipose tissues and is strikingly upregulated during adipogenesis in mice (Matsusue et al., 2008; Nishino et al., 2008; Toh et al., 2008). Cell death-inducing DNA fragmentation factor- $\alpha$-like effector $\mathrm{C}$ has the ability to enhance neutral lipid accumulation not only in adipocytes but also in many nonlipogenic cells (Puri et al., 2007). In mice, CIDEC overexpression leads to promotion of the formation of intracellular lipid droplets through TAG accumulation in hepatocytes in vitro 
and in vivo, whereas knockdown of CIDEC results in loss of hepatic lipids (Matsusue et al., 2008). In 3T3-L1 adipocytes, CIDEC depletion significantly stimulates lipolysis and reduces the size of lipid droplets (Keller et al., 2008). The mammary gland is one of the major lipid-synthesizing organs in dairy cows. However, little is known about the effect of CIDEC on milk lipid synthesis in the mammary gland of dairy cows.

Increased milking frequency in dairy cows results in greater milk production (Hillerton et al., 1990). Microarray analysis of the bovine mammary gland has shown that CIDEC expression is upregulated by increased milking frequency (Connor et al., 2008). This result suggests that CIDEC may participate in milk lipid synthesis. In mice and human adipocytes, CIDEC localizes to lipid droplets (Puri et al., 2007). However, no study directly investigating CIDEC expression in mammary gland of lactating cows has been reported. Peroxisome proliferator-activated receptor- $\gamma$ (PPAR $\gamma$ ) belongs to the nuclear receptor superfamily and is expressed in numerous types of cells, including adipocytes, macrophages, and mammary epithelial cells (Kang et al., 2015). In bovine and dairy goat mammary glands, PPAR $\gamma$ is considered to be a central regulator of lipid metabolism (Bionaz and Loor, 2008b; Kang et al., 2015). Gene network analysis in bovine mammary tissue shows that expression of PPAR $\gamma$ and its putative target genes is upregulated during lactation, suggesting a role for this nuclear receptor in the regulation of milk lipid synthesis (Bionaz and Loor, 2008b). The $C I D E C$ has been identified as a direct target gene of $\operatorname{PPAR} \gamma$, and its upregulation can elevate TAG levels in mouse hepatocyte (Matsusue et al., 2008). Whether CIDEC participates in regulating milk lipid synthesis in lactating dairy cow mammary gland has not been well established.

The objective of this study was to reveal the specific role of CIDEC in regulating milk lipid synthesis in the mammary gland of dairy cows. To meet this objective, 6 lactating Holstein cows in the third parity were allocated to high-fat milk (milk yield $33.9 \pm 2.1 \mathrm{~kg} / \mathrm{d}$, milk fat $>3.5 \%, \mathrm{n}=3$ ) and low-fat milk (milk yield $33.7 \pm$ $0.5 \mathrm{~kg} / \mathrm{d}$, milk fat $<3.5 \%, \mathrm{n}=3$ ) groups. Lactating cows were slaughtered at $90 \mathrm{DIM}$ and mammary tissues were collected to detect CIDEC expression. To evaluate the role of CIDEC in mediating milk lipid synthesis in the dairy cow mammary gland, mammary epithelial cells were isolated from lactating mammary tissues of dairy cows with high-fat milk. The expression of genes encoding enzymes and transcription factors involved in milk lipid synthesis were examined in cultured mammary epithelial cells by quantitative real-time PCR (qPCR) when $C I D E C$ was knocked down or overexpressed.

\section{MATERIALS AND METHODS}

\section{Animals and Tissue Collection}

The Northeast Agricultural University Animal Care and Use Committee (Harbin, China) approved all procedures involving dairy cows. Six multiparous Holstein cows (in the third parity, calving at 52 to 54 mo of age) in early lactation were used in this study. Amplification of the polymorphism DGAT1 K232A by tetra-primer amplification refractory mutation system PCR (Steinberg et al., 2009) showed that all cows carried the AA genotype of DGAT1 (Supplemental Figure S1; https:// doi.org/10.3168/jds.2016-11549). All animals were offered grass silage ad libitum supplemented with concentrates. Animals had access to a constant supply of fresh water and were milked twice daily at 0800 and $1530 \mathrm{~h}$.

Lactating cows were allocated to 2 groups (3 cows per group), the high-fat milk (milk fat $>3.5 \%$ ) and lowfat milk (milk fat $<3.5 \%$, milk fat decreased likely due to diet-induced milk fat depression) groups, according to their milk fat content (Table 1). Milk yield of lactating cows with high-fat milk was $33.9 \pm 2.1 \mathrm{~kg} / \mathrm{d}$. Milk yield of lactating cows with low-fat milk was $33.7 \pm$ $0.5 \mathrm{~kg} / \mathrm{d}$. Somatic cell count was $<50,000$ cells $/ \mathrm{mL}$ for all cows. The 6 lactating cows were slaughtered at 90 DIM. Immediately after exsanguination, several parenchyma samples were aseptically removed from the core of the mammary gland. Tissue samples for quantitation of protein and RNA were frozen immediately in liquid nitrogen and stored at $-80^{\circ} \mathrm{C}$ until assay. Mammary tissues for cell culture were collected in $50-\mathrm{mL}$ conical centrifuge tubes with Hanks' Balanced Salt Solution (14170, Life Technologies Corporation, Grand Island, $\mathrm{NY}$ ), transported, and preserved at $4^{\circ} \mathrm{C}$ until cell isolation and culture.

Table 1. Milk components of lactating Holstein dairy cows

\begin{tabular}{lcc}
\hline Milk component & Dairy cows with high-fat milk & Dairy cows with low-fat milk \\
\hline Milk protein (\%) & $3.27 \pm 0.04$ & $2.89 \pm 0.02$ \\
Milk fat (\%) & $4.17 \pm 0.01$ & $3.20 \pm 0.06$ \\
Lactose (\%) & $4.84 \pm 0.03$ & $4.52 \pm 0.09$ \\
DM (\%) & $11.93 \pm 0.01$ & $10.89 \pm 0.03$ \\
\hline
\end{tabular}




\section{Mammary Epithelial Cell Culture}

Primary cell culture from lactating mammary parenchymal tissues of 3 dairy cows with high-fat milk was performed as described (Hou et al., 2016). Briefly, the tissue samples were minced using surgical scissors. Minced samples were digested with collagenase III (C8490, Solarbio, Beijing, China) for $2 \mathrm{~h}$ at $37^{\circ} \mathrm{C}$. The digest was filtered through a nylon mesh and the filtrate centrifuged at $150 \times g$ for $10 \mathrm{~min}$ at room temperature. The cell inoculum was plated in Dulbecco's modified Eagle's medium:Nutrient Mixture F-12 (12400, Life Technologies, Carlsbad, CA) supplemented with 10\% fetal bovine serum (04-001-1ACS, BI Biological Industries, Kibbutz Beit-Haemek, Israel), $100 \mathrm{U} / \mathrm{mL}$ of penicillin, $100 \mu \mathrm{g} / \mathrm{mL}$ of streptomycin and lactating hormones $(5 \mu \mathrm{g} / \mathrm{mL}$ insulin, $1 \mu \mathrm{g} / \mathrm{mL}$ of prolactin, and $1 \mu \mathrm{g} / \mathrm{mL}$ of hydrocortisone; Sigma-Aldrich, St. Louis, $\mathrm{MO})$ in cell culture flasks coated with $0.5 \%$ rat tail collagen (Sigma-Aldrich) and incubated in 5\% $\mathrm{CO}_{2}$ at $37^{\circ} \mathrm{C}$. The primary cells were trypsinized at $\sim 80 \%$ confluency and passaged. Pure mammary epithelial cells were obtained after 3 passages. Immunofluorescence was used to detect cytokeratin 18 expression in the cultured cells to confirm that the isolated cells were purified mammary epithelial cells (Supplemental Figure 2A; https://doi.org/10.3168/jds.2016-11549). All experiments were conducted using cells within passage 10. Lipid droplets and $\beta$-casein expression were detected by immunofluorescence before experiments to ensure that the cells we used were capable of synthesizing milk components (Supplemental Figures 2B and 2C; https://doi.org/10.3168/jds.2016-11549).

\section{Plasmid Construction}

Total RNA from lactating mammary tissue samples was extracted using TRIzol reagent (15596, Life Technologies). The RNA quantity and purity were determined by a NanoDrop 2000c spectrophotometer (Thermo Scientific, Wilmington, DE). The RNA integrity was analyzed by denaturing agarose gel electrophoresis with the $28 \mathrm{~S}$ rRNA band appearing approximately twice as intense as the 18S rRNA band. Total RNA was reverse transcribed to cDNA using Moloney murine leukemia virus reverse transcriptase (C28025, Life Technologies) with oligo dT at $37^{\circ} \mathrm{C}$ for $50 \mathrm{~min}$. To construct the CIDEC expression plasmid, full-length bovine $C I D E C$ was amplified from mammary tissue cDNA using PCR and subcloned into the BamH I/ Hind III sites of the pcDNA3.1 (+) vector (Invitrogen, Carlsbad, CA). The following PCR primer pair was used: forward $=$ 5'-CCCAAGCTTATGGAATACGC-
CATGAAGTCCCTCAGC-3'; reverse $=5^{\prime}$-CGCGGATCCTCACTGCAGCATCTTCAGACTG-

GTTG-3'. The integrity of plasmid DNA was verified by DNA sequencing.

To construct the short hairpin RNA (shRNA) plasmid for targeting CIDEC, a pair of oligonucleotides specific for bovine $C I D E C$ sequence and a pair of nontargeting oligonucleotides (scramble) were chemically synthesized and subcloned into the BamHI/HindIII sites of pSilencer 3.1-H1 (Ambion, Carlsbad, CA). The shRNA sequences used in this study were as follows: CIDEC, forward $=5$ '-GATCCGCACATACTCCGTCTCCTATTCAAGAGATAGGAGACGGAGTATGTGCTTTTTTGGAAA-3'; reverse $=5$-AGCTTTTCCAAAAAAGCACATACTCCGTCTCCTATCTCTTGAATAGGAGACGGAG-

TATGTGCG-3'. Scramble, forward = 5'-GATCCGCACAAGCTGGAGTACAACTTCAAGAGAGTTG-

TACTCCAGCTTGTGCTTTTTTGGAAA-3'; reverse $=$ 5'-AGCTTTTCCAAAAAAGCACAAGCTGGAGTACAACTCTCTTGAAGTTGTACTCCAGCTTGTGCG-3'.

\section{Transfection}

Transient transfection was performed using Lipofectamine 2000 (11668-019, Life Technologies) according to the manufacturer's instructions (Hou et al., 2016). Briefly, mammary epithelial cells were plated in 6-well plates at $1 \times 10^{6}$ cells/well. For CIDEC knockdown, $\sim 80 \%$ confluent cells were transfected with either $2.5 \mu \mathrm{g}$ of $C I D E C$-specific shRNA or nontargeting shRNA as a negative control. For $C I D E C$ overexpression, cells were transfected with either $2.5 \mu \mathrm{g}$ of pcDNA3.1-CIDEC or pcDNA3.1(+) as a negative control. All experiments were performed in triplicate. After $24 \mathrm{~h}$ of transfection, cells were harvested for qPCR assay. After $48 \mathrm{~h}$ of transfection, cells were harvested for lipid droplet immunostaining and TAG content assays.

\section{Western Blot Analysis}

Cultured mammary epithelial cells were lysed with RIPA buffer (P0013C, Beyotime Biotechnology, Shanghai, China) containing $0.5 \mathrm{mM}$ PMSF (ST506, Beyotime Biotechnology), $5 \mu \mathrm{g} / \mathrm{mL}$ of aprotinin (A1153, Sigma-Aldrich), and $5 \mu \mathrm{g} / \mathrm{mL}$ of leupeptin (L2884, Sigma-Aldrich), and quantitated by a BCA protein assay kit (23227, Thermo Fisher Scientific Inc., Waltham, MA). Equal amounts of protein $(30 \mu \mathrm{g})$ were separated on a 10\% SDS-polyacrylamide gel and blotted onto a nitrocellulose membrane. The membrane was blocked for $1 \mathrm{~h}$ with $5 \%$ skim milk in Tris-buffered saline with 
$0.1 \%$ (vol/vol) Tween-20 and incubated overnight at $4^{\circ} \mathrm{C}$ with antibody specific to CIDEC (1:500 dilution, ab198204, Abcam, Cambridge, MA) or $\beta$-actin (1:1,000 dilution, \#4970, Cell Signaling Technology, Beverly, MA). The blot was then treated with horseradish peroxidase-conjugated anti-rabbit IgG (1:2000 dilution, ZB-2301, ZSGB-BIO, Beijing, China) for $1 \mathrm{~h}$. Proteins were visualized using enhanced chemiluminescence detection reagent (6683, Cell Signaling Technology). Densitometry analysis was performed with Image-Pro Plus 6.0 (Media Cybernetics Inc., Warrendale, PA) and target protein expression was normalized to $\beta$-actin expression.

\section{Immunostaining and Confocal Microscopy}

For determination of CIDEC localization in mammary tissues and cultured mammary epithelial cells, $8 \mu \mathrm{m}$ thick cryosections or cultured mammary epithelial cells were fixed in $4 \%$ paraformaldehyde for $20 \mathrm{~min}$ at room temperature, permeabilized with $0.05 \%$ Triton X-100 (vol/vol) in PBS, blocked with $10 \%$ goat serum in PBS for $1 \mathrm{~h}$ at $37^{\circ} \mathrm{C}$, and treated overnight at $4^{\circ} \mathrm{C}$ with antiCIDEC (1:100 dilution, ab198204, Abcam). Sections or cells were washed 3 times with PBS and incubated with tetraethyl rhodamine isothiocyanate-conjugated goat anti-rabbit IgG (1:200 dilution, ZF-0316, ZSGB-BIO) for $30 \mathrm{~min}$ at $37^{\circ} \mathrm{C}$. Following 3 washes, sections or cells were stained with Bodipy 493/503 (1:1,000 dilution, D3922, stock concentration $1 \mathrm{mg} / \mathrm{mL}$, Invitrogen) for lipid droplet staining, and nuclei were stained with 4',6-diamidino-2-phenylindole. After extensive washing, sections or cells were mounted in 1,4-diazabicyclooctane triethylenediamine. Fluorescence was observed by confocal microscopy (TCS SP2, Leica Microsystems GmbH, Wetzlar, Germany).

For detection of lipid droplets in CIDEC knockdown or overexpressed cells, mammary epithelial cells plated on coverslips were washed twice with PBS and fixed in $4 \%$ paraformaldehyde for $20 \mathrm{~min}$ at room temperature. After 3 washes with PBS, cells were stained for $20 \mathrm{~min}$ at $37^{\circ} \mathrm{C}$ with Bodipy 493/503 (1:1,000 dilution, D3922, Invitrogen) for lipid droplets, following by incubation for $10 \mathrm{~min}$ with $4^{\prime}, 6$-diamidino-2-phenylindole for nuclei staining. Cells were washed 3 times with PBS and mounted in 1,4-diazabicyclooctane triethylenediamine. Fluorescence was observed by confocal microscopy (TCS SP2, Leica Microsystems GmbH).

\section{Measurement of TAG Content}

Mammary epithelial cells were washed 3 times with pre-warmed PBS and then incubated in serum-free me- dium containing $1 \%$ fatty acid-free BSA (A8806, Sigma-Aldrich) for $24 \mathrm{~h}$ before TAG content assay. For cellular TAG content determination, lipids were extracted from cultured mammary epithelial cells with hexane/ isopropanol $(3: 2, \mathrm{vol} / \mathrm{vol})$. The lipids were dried with nitrogen and dissolved in ethanol. The TAG levels were detected by a TAG assay kit (E1013, Applygen Technologies Inc., Beijing, China). Protein concentrations in the samples were measured by a BCA protein assay kit (23227, Thermo Fisher Scientific Inc.), and TAG levels were normalized to the protein concentration of each sample. Experiments were performed in triplicate.

\section{qPCR}

Total RNA was isolated from cultured mammary epithelial cells with TRIzol reagent (15596, Life Technologies) according to the manufacturer's instructions (Hou et al., 2016). The RNA concentrations were measured on a NanoDrop 2000c spectrophotometer (Thermo Scientific). Total RNA was reverse-transcribed to cDNA with M-MLV reverse transcriptase (28025, Life Technologies). Oligo dT was used as the primer for reverse transcription. Expression levels of selected genes involved in milk lipid synthesis were determined by qPCR analysis using gene-specific primer sets (Table 2). Primers were designed using Primer Premier 5.0 (Premier Biosoft, Palo Alto, CA). Real-time qPCR was performed using the SYBR Premix Ex Taq (RR420A, TaKaRa Biotechnology Co., Ltd., Dalian, China) in the Applied Biosystems 7500 Real-Time PCR system (Applied Biosystems, Grand Island, NY). Thermal cycling conditions were: $30 \mathrm{~s}$ of predenaturalization at $95^{\circ} \mathrm{C}$, followed by 40 cycles of $5 \mathrm{~s}$ denaturation at $95^{\circ} \mathrm{C}$ and 34 $\mathrm{s}$ annealing and extension at $60^{\circ} \mathrm{C}$. The PCR product specificity was verified by postamplification melting curve analysis and by running products on an agarose gel. Each gene was amplified in a separate reaction and each reaction was performed in triplicate. The abundance of $\beta$-actin was not significantly different between treatments; therefore, the relative expression of target genes was normalized to $\beta$-actin and calculated by the $2^{-\Delta \Delta \mathrm{CT}}$ method (Schmittgen and Livak, 2008).

\section{Statistical Analysis}

Results are presented as the mean \pm SEM. Data from 3 independent experiments were subjected to statistical analysis using Prism 6 software (GraphPad, La Jolla, CA). The effect of CIDEC knockdown or overexpression on transcript abundance of genes encoding enzymes and transcription factors involved in milk lipid synthesis in bovine mammary epithelial cells was 
Table 2. Primer sequences used for quantitative real-time $\mathrm{PCR}^{1}$

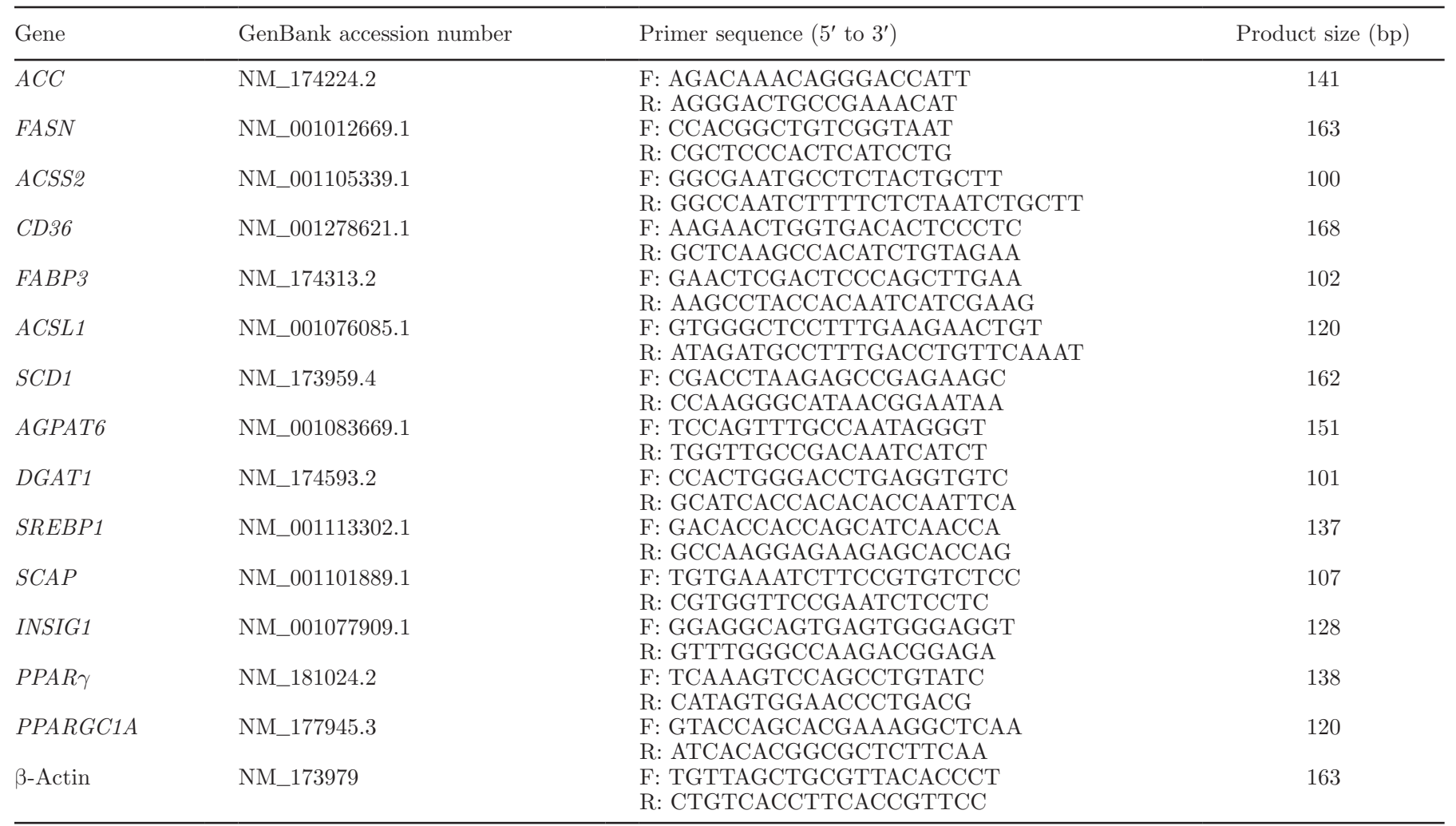

${ }^{1} A C C=$ acetyl-CoA carboxylase; $F A S N=$ fatty acid synthase; $A C S S 2=$ acyl-CoA synthetase short-chain family member $2 ; C D 36=$ cluster of differentiation 36 (fatty acid translocase); FABP $3=$ fatty acid binding protein $3 ; A C S L 1=$ acyl-CoA synthetase long-chain family member 1: $S C D 1=$ stearoyl-CoA desaturase 1; $A G P A T 6=1$-acylglycerol-3-phosphate-O-acyltransferase $6 ; D G A T 1=$ diacylglycerol acyltransferase 1; $S R E B P 1=$ sterol regulatory element-binding protein $1 ; S C A P=\mathrm{SREBP}$ cleavage activating protein; INSIG1 = insulin-induced gene 1; PPAR $\gamma$ $=$ peroxisome proliferator-activated receptor- $\gamma ; P P A R G C 1 A=$ peroxisome proliferator-activated receptor gamma coactivator-1 $\alpha$. $\mathrm{F}=$ forward; $\mathrm{R}=$ reverse.

assessed using a mixed model ANOVA. Fixed effect in the model was treatment, whereas the random effect was replicate. Bonferroni's multiple comparison test was used for post-ANOVA comparisons. Differences at $P<0.05$ were considered significant.

\section{RESULTS}

\section{CIDEC Expression in Lactating Mammary Glands of Dairy Cows}

To determine the tissue-specific expression of CIDEC in lactating cows, we detected CIDEC expression in lactating mammary tissues with high- and low-fat milk. Immunofluorescence staining of sections of lactating mammary glands with high- and low-fat milk (diet-induced milk fat depression) both showed that mammary epithelial cells contained cytoplasmic lipid droplets, most of which were located near the lumen face. As indicated by the images in Figure 1A, CIDEC was mainly observed in the cytoplasm of epithelial cells and localized to lipid droplets. Furthermore, lipid droplets and CIDEC protein were also detected in isolated lactating mammary epithelial cells. In addition to its previously established localization on lipid droplets, a substantial portion of CIDEC protein was detected in the nucleus (Figure 1B), suggesting that CIDEC may participate in regulation of milk lipid synthesis.

\section{Effect of CIDEC on Milk Lipid Synthesis in Mammary Glands of Dairy Cows}

To investigate whether CIDEC has any role in regulating milk lipid synthesis in lactating mammary glands of dairy cows, cultured mammary epithelial cells were transiently transfected either with shRNA directed against $C I D E C$ or scrambled shRNA as a negative control. As shown in Figures 2A and 2B, the mRNA and protein levels of CIDEC were significantly decreased when cells were transfected with $C I D E C$-specific shRNA $(P<0.01$; Figures $2 \mathrm{~A}$ and $2 \mathrm{~B})$, indicating the effectiveness of shRNA in reducing CIDEC mRNA and 
protein abundance. The cellular TAG levels in CIDECknockdown mammary epithelial cells were reduced $(P$ $<0.05$; Figure 2C). Consistent with this, the population of lipid droplets was decreased in $C I D E C$-knockdown cells compared with cells transfected with scrambled shRNA (Figure 2D).

Next, we treated cultured mammary epithelial cells with the pcDNA3.1-CIDEC vector or empty pcDNA3.1(+) vector as a negative control. Compared with cells carrying the empty pcDNA3.1(+) vector, the mRNA and protein levels of CIDEC in dairy cow mammary epithelial cells were significantly increased by transfection with pcDNA3.1-CIDEC vector $(P<0.01$; Figures $3 \mathrm{~A}$ and $3 \mathrm{~B}$ ). The overexpression of CIDEC in mammary epithelial cells markedly enhanced TAG synthesis $(P<0.01$; Figure 3C). Control cells transfected with the empty pcDNA3.1(+) vector exhibited smaller lipid droplets. In contrast, transfection of mammary epithelial cells with pcDNA3.1-CIDEC resulted in lipid droplets with increased size (Figure 3D). Overall, these data support the notion that CIDEC is crucial in

A

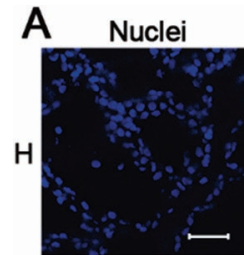

CIDEC
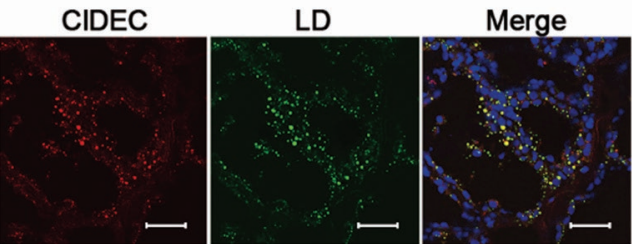

$\mathrm{L}$
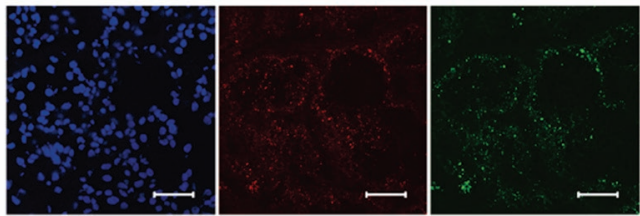

B

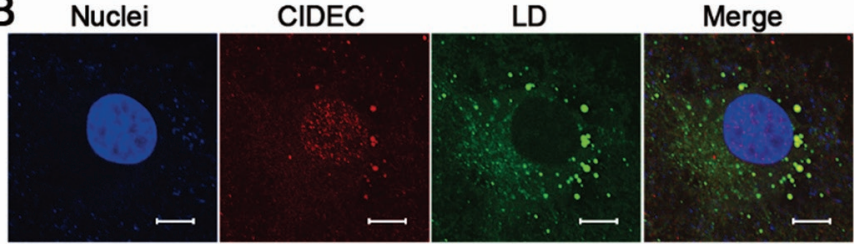

Figure 1. The expression of cell death-inducing DNA fragmentation factor- $\alpha$-like effector $\mathrm{C}(C I D E C)$ in lactating mammary tissues and cultured mammary epithelial cells of dairy cows. (A) Immunofluorescence staining showing the localization of CIDEC expression in mammary tissues of dairy cows. Scale bar $=45 \mu \mathrm{m} ; \mathrm{H}=$ lactating mammary tissues with high-fat milk; $\mathrm{L}=$ lactating mammary tissues with lowfat milk. (B) Immunofluorescence staining showing the localization of $C I D E C$ expression in cultured mammary epithelial cells of dairy cows. Scale bar $=10 \mu \mathrm{m}$. For A and B, CIDEC was labeled by tetraethyl rhodamine isothiocyanate (red, light gray in the CIDEC and Merge panels in the grayscale version), lipid droplets (LD) were labeled by Bodipy 493/503 (green, light gray in the LD and Merge panels in the grayscale version; Invitrogen, Carlsbad, CA), and nuclei were labeled by $4^{\prime}, 6$-diamidino-2-phenylindole (blue, dark gray in the Nuclei and Merge panels in the grayscale version). Merge images show the overlap of nuclei, CIDEC, and lipid droplets. Color version available online.

controlling milk lipid synthesis in mammary glands of dairy cows.

\section{Effects of CIDEC on Expression of Enzymes Involved in Milk Lipid Synthesis}

To explore the molecular events by which CIDEC mediates milk lipid synthesis, we conducted an analysis
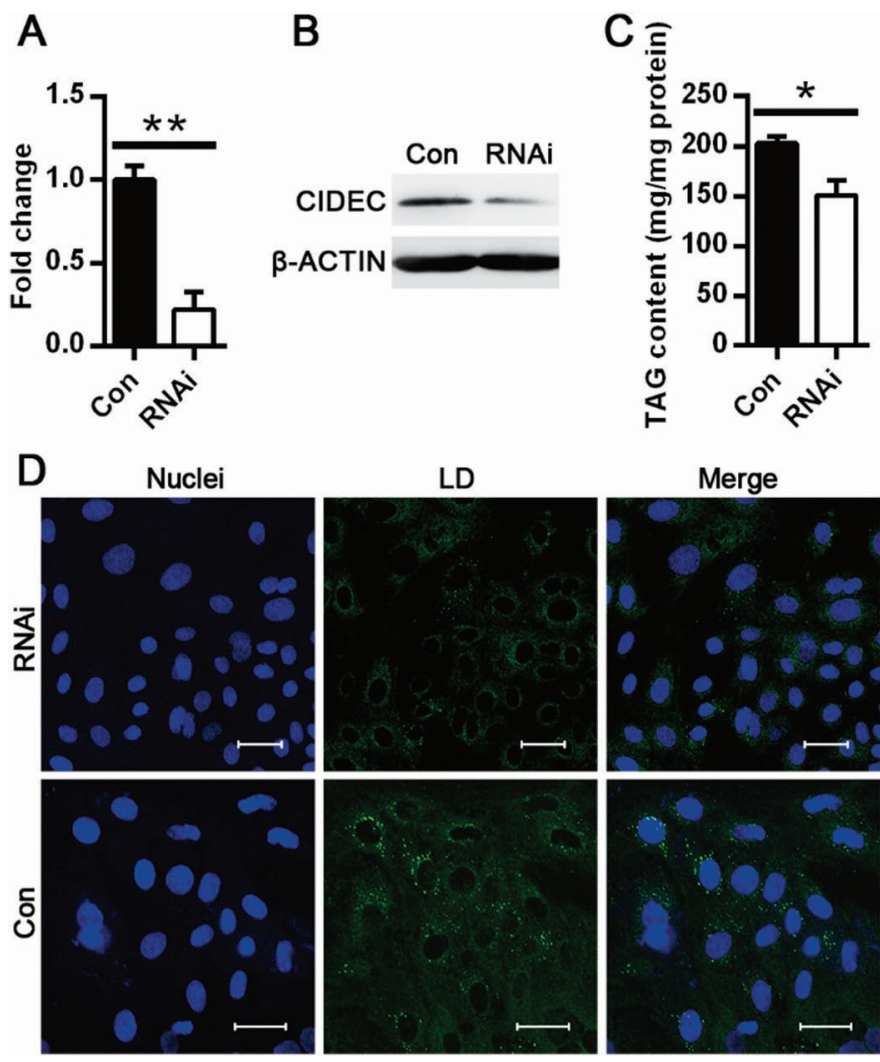

Figure 2. The effects of cell death-inducing DNA fragmentation factor- $\alpha$-like effector $\mathrm{C}(C I D E C)$ knockdown on milk lipid synthesis. (A) Quantitative real-time PCR (qPCR) analyses of the mRNA levels of $C I D E C$ in $C I D E C$-knockdown (RNAi) and scrambled short hairpin RNA (shRNA) control (Con) mammary epithelial cells. The RNA was extracted from control and shRNA-treated cells, and mRNA levels were measured by qPCR and normalized to $\beta$-actin mRNA. (B) Western blot analyses of the protein levels of CIDEC and $\beta$-actin (loading control) in CIDEC-knockdown (RNAi) and scrambled shRNA control (Con) mammary epithelial cells. Protein lysates from control and CIDEC shRNA-treated cells were loaded at $30 \mu \mathrm{g} /$ lane and probed with antibody against CIDEC or $\beta$-actin. (C) Knockdown of $C I D E C$ in cultured mammary epithelial cells of dairy cows (RNAi) decreased triacylglycerol (TAG) content. (D) Immunofluorescence staining showing the lipid droplets in $C I D E C$-knockdown (RNAi) and scrambled shRNA control (Con) mammary epithelial cells. Lipid droplets (LD) were labeled by Bodipy 493/503 (green, light gray in the LD and Merge panels in the grayscale version; Invitrogen, Carlsbad, CA), and nuclei were labeled by 4',6-diamidino-2-phenylindole (blue, dark gray in the Nuclei and Merge panels in the grayscale version). Merge images show the overlap between nuclei and lipid droplets. Scale bar $=30 \mu \mathrm{m}$. Results are shown as the mean $\pm \mathrm{SEM}$ from 3 independent experiments. ${ }^{*} P<0.05$; ${ }^{*} * P<0.01$. Color version available online. 
of gene expression in lactating mammary epithelial cells when $C I D E C$ was knocked down or overexpressed. The mRNA expression of acyl-CoA synthetase short-chain family member 2 (ACSS2), acetyl-CoA carboxylase (ACC), and fatty acid synthase (FASN), which encode enzymes involved in the de novo FA synthesis (Bionaz

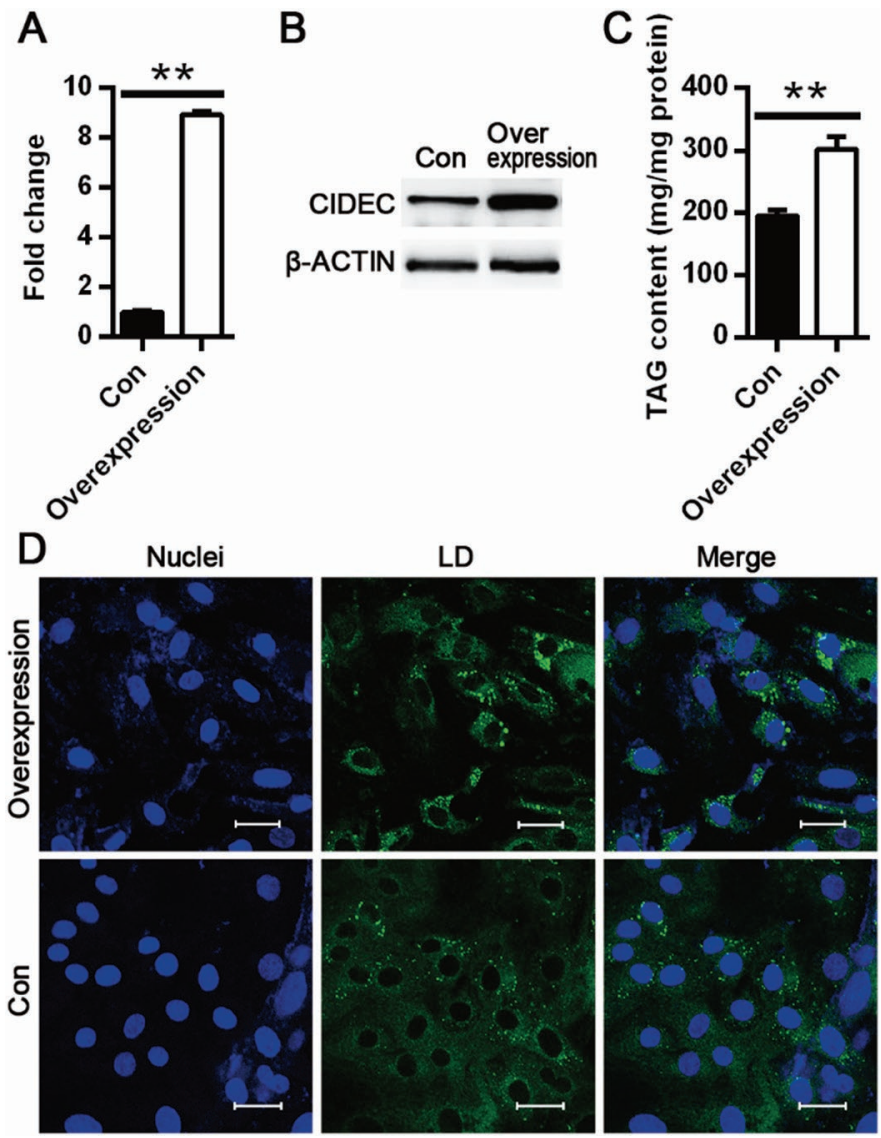

Figure 3. The effects of cell death-inducing DNA fragmentation factor- $\alpha$-like effector $\mathrm{C}(C I D E C)$ overexpression on milk lipid synthesis. (A) Quantitative real-time PCR (qPCR) analyses of the mRNA levels of $C I D E C$ in $C I D E C$-overexpressing mammary epithelial cells. The RNA was extracted from control (Con) and CIDEC-overexpressing cells, and mRNA levels were measured by qPCR and normalized to $\beta$-actin mRNA. (B) Western blot analyses of the protein levels of CIDEC and $\beta$-actin (loading control) in CIDEC-overexpressing mammary epithelial cells. Protein lysates from control and CIDEC-overexpressing cells were loaded at $30 \mu \mathrm{g} /$ lane and probed with antibody against CIDEC or $\beta$-actin. (C) Overexpression of CIDEC in cultured mammary epithelial cells of dairy cows increased triacylglycerol (TAG) content. (D) Immunofluorescence staining showing the lipid droplets (LD) in CIDEC-overexpressing mammary epithelial cells. Lipid droplets were labeled by Bodipy 493/503 (green, light gray in the LD and Merge panels in the grayscale version; Invitrogen, Carlsbad, CA), and nuclei were labeled by $4^{\prime}, 6$-diamidino-2-phenylindole (blue, dark gray in the Nuclei and Merge panels in the grayscale version). Merge images show the overlap between nuclei and lipid droplets. Scale bar $=30 \mu \mathrm{m}$. In the control group (Con), cells were transfected with the pcDNA3.1(+) vector. In the overexpression group, cells were transfected with the pcDNA3.1-CIDEC vector. Results are shown as the mean \pm SEM from 3 independent experiments. ${ }^{* *} P<0.01$. Color version available online.
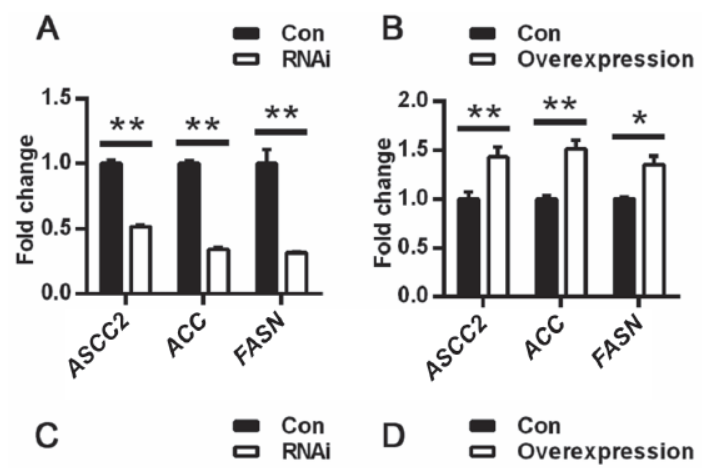

D Con
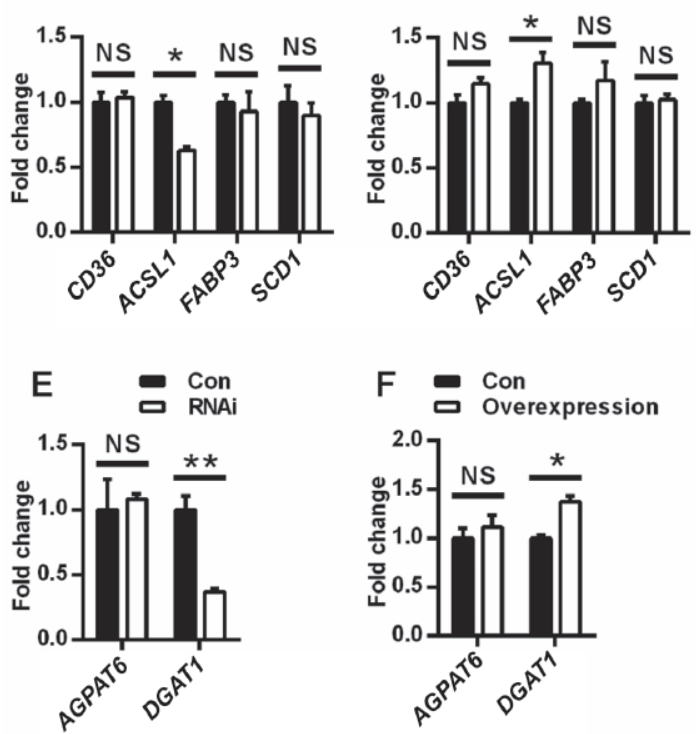

Figure 4. Effects of cell death-inducing DNA fragmentation factor$\alpha$-like effector C (CIDEC) on expression of genes encoding enzymes involved in milk lipid synthesis. (A) The mRNA abundance of genes encoding enzymes involved in de novo fatty acid synthesis in mammary epithelial cells with $C I D E C$ knockdown (RNAi). (B) The mRNA abundance of genes encoding enzymes involved in de novo fatty acid synthesis in mammary epithelial cells with CIDEC overexpression. (C) The mRNA abundance of genes encoding enzymes involved in fatty acid uptake, activation, transportation, and desaturation in mammary epithelial cells with CIDEC knockdown (RNAi). (D) The mRNA abundance of genes encoding enzymes involved in fatty acid uptake, activation, transportation, and desaturation in mammary epithelial cells with CIDEC overexpression. (E) The mRNA abundance of genes encoding enzymes involved in triacylglycerol (TAG) synthesis in mammary epithelial cells with $C I D E C$ knockdown (RNAi). (F) The mRNA abundance of genes encoding enzymes involved in TAG synthesis in mammary epithelial cells with $C I D E C$ overexpression. For A, C, and E, total RNA was extracted from mammary epithelial cells transfected with $C I D E C$ short hairpin RNA (RNAi) or scrambled RNA as a negative control (Con) for $24 \mathrm{~h}$. For B, D, and F, total RNA was extracted from mammary epithelial cells transfected with pcDNA3.1-CIDEC or pcDNA3.1(+) vector as control (Con) for $24 \mathrm{~h}$. The mRNA levels were measured by quantitative real-time PCR and normalized to $\beta$-actin mRNA. Results are shown as the mean \pm SEM from 3 independent experiments, each of which was performed in triplicate. ${ }^{*} P<0.05$; ${ }^{*} P<0.01 . A C S S 2=$ acyl-CoA synthetase short-chain family member 2; $A C C=$ acetyl-CoA carboxylase; $F A S N=$ fatty acid synthase; $C D 36=$ cluster of differentiation 36 (fatty acid translocase); ACSL1 $=$ acyl-CoA synthetase long-chain family member $1 ; F A B P 3=$ fatty acid binding protein $3 ; S C D 1=$ stearoyl-CoA desaturase $1 ; A G P A T 6$ = 1-acylglycerol-3-phosphate-O-acyltransferase $6 ; D G A T 1=$ diacylglycerol acyltransferase 1 . 
and Loor, 2008b), was reduced by $\sim 50,60$, and $65 \%$, respectively, by CIDEC shRNA treatment compared with the control (Figure 4A). In contrast, the overexpression of $C I D E C$ in dairy cow mammary epithelial cells significantly increased the levels of ACSS2 $(P<$ $0.01)$, ACC $(P<0.01)$, and FASN $(P<0.05$; Figure 4B) mRNA.

The mRNA expression of acyl-CoA synthetase long-chain family member 1 (ACSL1), which encodes an enzyme involved in long-chain FA activation, was decreased by $\sim 30 \%$ when cells were transfected with CIDEC shRNA (Figure 4C). Overexpression of CIDEC in the cells significantly increased ACSL1 mRNA levels $(P<0.05$; Figure 4D). However, compared with the control, the expression levels of the genes for FA translocator FAT/CD36 (CD36), fatty acid binding protein 3 (FABP3), and stearoyl-CoA desaturase 1 (SCD1), which are involved in long-chain FA uptake from blood, intracellular FA trafficking, and desaturation, were not changed in CIDEC-knockdown cells and in cells overexpressing CIDEC (Figures $4 \mathrm{C}$ and $4 \mathrm{D}$ ).

To evaluate the effect of CIDEC on the expression of enzymes involved in TAG synthesis, we analyzed the mRNA levels of 1-acylglycerol-3-phosphate-O-acyltransferase 6 (AGPAT6), and diacylglycerol acyltransferase 1 (DGAT1). The results showed that knockdown of CIDEC in mammary epithelial cells led to substantially lower expression of DGAT1 $(-60 \%, P<0.01)$, but not of $A G P A T 6$. In contrast, the overexpression of $C I D E C$ resulted in higher DGAT1 expression $(P<$ 0.05 ) but similar levels of expression of AGPAT6 (Figures $4 \mathrm{E}$ and $4 \mathrm{~F}$ ).

\section{Effects of CIDEC on Expression of Transcription Factors Involved in Milk Lipid Synthesis}

Several transcription factors regulate milk lipid synthesis in dairy cows. The mRNA expression of sterol regulatory element-binding protein 1 (SREBP1) and SREBP cleavage activating protein (SCAP) were reduced by $\sim 45$ and $66 \%$, respectively, by $C I D E C$ shRNA treatment compared with control $(P<0.01$; Figure $5 \mathrm{~A})$. In contrast, the overexpression of $C I D E C$ in dairy cow mammary epithelial cells significantly increased SREBP1 and SCAP expression levels $(P<0.05$; Figure 5B). However, compared with the control, the expression of insulin-induced gene 1 (INSIG1), which interacts with SCAP and regulates the responsiveness of SREBP1 processing via SCAP, was not changed in $C I D E C$-knockdown cells or in cells overexpressing $C I$ $D E C$ (Figures 5A and 5B). Moreover, the knockdown or overexpression of $C I D E C$ did not affect the expression levels of PPAR $\gamma$ and its coactivator, peroxisome
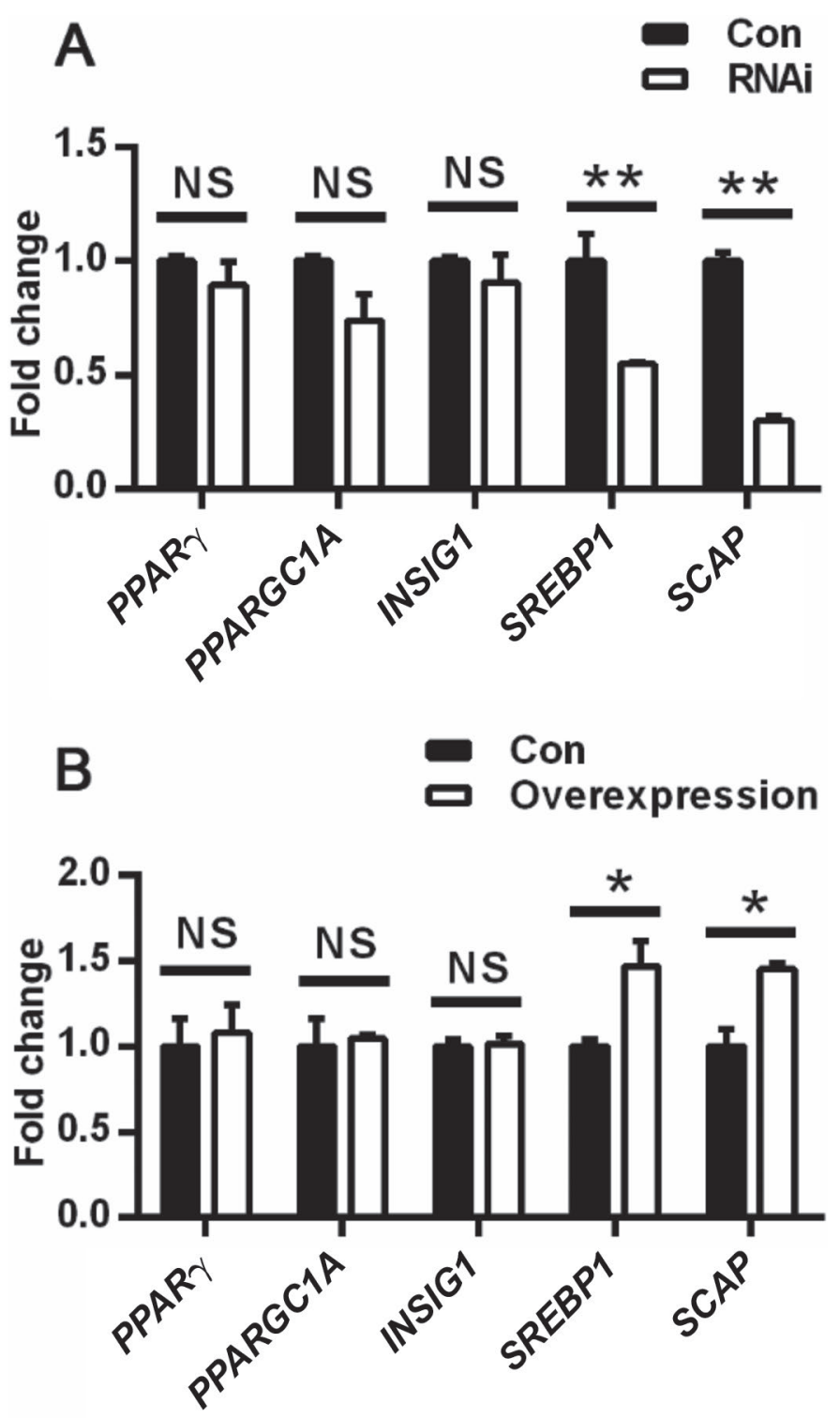

Figure 5. Effects of cell death-inducing DNA fragmentation factor$\alpha$-like effector $\mathrm{C}$ (CIDEC) on expression of genes encoding transcription factors involved in milk lipid synthesis. (A) The mRNA abundance of genes encoding transcription factors involved in milk lipid synthesis in mammary epithelial cells with CIDEC knockdown (RNAi). Total RNA was extracted from mammary epithelial cells transfected with $C I D E C$ short hairpin RNA (RNAi) or scrambled RNA as a negative control (Con) for $24 \mathrm{~h}$. The mRNA levels were measured by quantitative realtime PCR (qPCR) and normalized to $\beta$-actin mRNA. (B) The mRNA abundance of genes encoding transcription factors involved in milk lipid synthesis in mammary epithelial cells with CIDEC overexpression. Total RNA was extracted from mammary epithelial cells transfected with pcDNA3.1-CIDEC or pcDNA3.1(+) vector as the control (Con) for $24 \mathrm{~h}$. The mRNA levels were measured by $\mathrm{qPCR}$ and normalized to $\beta$-actin mRNA. Results are shown as the mean \pm SEM from 3 independent experiments, each of which was performed in triplicate. ${ }^{*} P<0.05 ;{ }^{* *} P<0.01$. PPAR $\gamma=$ peroxisome proliferator-activated receptor- $\gamma ; P P A R G C 1 A=$ peroxisome proliferator-activated receptor gamma coactivator-1 $\alpha$; INSIG1 = insulin-induced gene 1; SREBP1 = sterol regulatory element-binding protein $1 ; S C A P=$ SREBP cleavage activating protein. 
proliferator-activated receptor gamma coactivator- $1 \alpha$ (PPARGC1A; Figures 5A and 5B).

\section{DISCUSSION}

Milk lipid synthesis plays a central role in determining dairy product quality. Understanding the biosynthesis of milk lipids and the factors that influence milk lipid quantity and quality are important for the dairy industry. Cell death-inducing DNA fragmentation factor$\alpha$-like effector $\mathrm{C}$ is known to be crucial in promoting formation of large lipid droplets in brown and white adipocytes (Nishino et al., 2008; Toh et al., 2008). However, little is known about its expression and function in dairy cow mammary gland. Our study demonstrates that CIDEC is expressed in lactating mammary tissues and cultured mammary epithelial cells of dairy cows. We also observed CIDEC expression in dairy cow mammary tissues during diet-induced milk fat depression. The nuclear localization of CIDEC suggests that it participates in controlling milk lipid synthesis in lactating dairy cows.

In the current study, we observed that $C I D E C$ deficiency resulted in markedly reduced milk lipid content as well as reduction in the transcription of key genes involved in milk lipid synthesis. In contrast, the overexpression of CIDEC in mammary epithelial cells of dairy cows markedly increased the expression of key genes involved in milk lipid synthesis and increased milk lipid concentration. These data are in agreement with a previous report that forced expression of CIDEC by adenovirus in mouse hepatocytes leads to increased TAG levels, whereas knockdown by adenovirus expression of $C I D E C$ shRNA results in lower accumulation of hepatic TAG compared with control adenovirus-infected liver (Matsusue et al., 2008).

In cows, about half of the FA, including short-chain FA (4-8 carbons), medium-chain FA (10-14 carbons), and a portion of 16-carbon FA, come from de novo FA synthesis (Bauman and Griinari, 2003). As the precursor for de novo FA synthesis, acetate needs to be activated by acyl-CoA synthetase to produce acetylCoA (Ma and Corl, 2012). The ACSS2 is the isoform responsible for acetate activation during de novo FA synthesis in dairy cow mammary gland (Bionaz and Loor, 2008b). The transcription pattern of ACSS2 corresponds with bovine mammary acetyl-CoA production throughout lactation (Mellenberger et al., 1973). In a sequence of steps, de novo synthesis is carried out by ACC and FASN utilizing acetyl-CoA. The ACC is the regulatory enzyme in FA synthesis as its activity is lower than other enzymes involved in lipogenesis and it possesses numerous kinetic properties characteristic of a regulatory enzyme (Mellenberger et al., 1973). The
FASN performs the majority of enzymatic steps of FA synthesis (Martin et al., 1961), and it is responsible for the production of short- and medium-chain FA as well as a substantial portion of long-chain FA (Smith, 2009). Gene expression studies have revealed that the activation of ACSS2, ACC, and FA synthesis in dairy cow mammary epithelium occurs in response to parturition and the onset of lactation (Bionaz and Loor, 2008b). In the current study, transcript abundance of $A C S S 2, A C C$, and FASN was decreased significantly with CIDEC shRNA, in agreement with TAG content data, indicating that de novo FA synthesis might be regulated by CIDEC in mammary epithelial cells of dairy cows.

Long-chain FA in milk lipids, including a portion of 16-carbon FA, are derived from the uptake of circulating lipids (Bauman and Griinari, 2003). It has been reported that CD36 is involved in FA uptake in bovine mammary cells, and its mRNA shows a large increase in expression during lactation (Bionaz and Loor, 2008b). The activation of long-chain FA is catalyzed by ACSL in the inner face of the plasma membrane before participating in TAG synthesis (Mashek and Coleman, 2006). The ACSL1 mRNA is predominant among ACSL isoforms in bovine mammary tissue (Bionaz and Loor, 2008a). Long-chain FA or long-chainacyl-CoA within cells can be captured by FABP, which transports them to specific intracellular organelles for utilization. The FABP3 is the most abundant isoform in lactating bovine mammary gland, and the important function of FABP3 in bovine mammary gland is to provide long-chain FA for SCD1 to produce UFA or to directly provide long-chain-acyl-CoA for TAG synthesis (Bionaz and Loor, 2008b). In this study, we observed a reduction in ACSL1 mRNA in mammary epithelial cells treated with CIDEC shRNA for $24 \mathrm{~h}$, indicating that long-chain FA activation is regulated transcriptionally by CIDEC. However, CD $36, F A B P 3$, and SCD1 expression were not affected by CIDEC knockdown or overexpression, suggesting that CIDEC is not involved in the transcriptional regulation of long-chain FA uptake, trafficking, and desaturation.

During milk lipid synthesis, AGPAT6 and DGAT1 are required for TAG production in bovine mammary gland (Bionaz and Loor, 2008a). The role of AGPAT6 is to add FA to the sn-2 position of acylglycerol-3-phosphate. When AGPAT6 is knocked out in lactating mice, they fail to synthesize milk lipids (Beigneux et al., 2006). In the bovine mammary gland, AGPAT6 expression is markedly upregulated during lactation (Bionaz and Loor, 2008a). In our study, although the expression of $S R E B P 1$ was increased with $C I D E C$ overexpression, AGPAT6 expression was not affected. This result is consistent with the report that AGPAT6 is not regu- 
lated by SREBP1 in bovine mammary epithelial cells (Ma and Corl, 2012). Diacylglycerol acyltransferase 1 is expressed in almost all tissues, including the mammary gland (Farese et al., 2000). Female DAGT1-deficient mice are unable to lactate (Smith et al., 2000). Diacylglycerol acyltransferase 1 catalyzes the final step in TAG synthesis. It has high affinity for butyryl-CoA and even higher affinity for palmitoyl-CoA (Marshall and Knudsen, 1979). In our study, the reduction in DGAT1 mRNA with CIDEC shRNA treatment indicates that DGAT1 is controlled by CIDEC to affect milk lipid synthesis.

Transcriptional regulation of TAG synthesis is mediated by several different factors. Sterol regulatory element-binding protein 1 is a transcription factor that controls genes involved in FA and TAG synthesis (Brown and Goldstein, 1997). In mouse hepatocytes, SREBP1 and its regulatory protein, SCAP, combine to regulate expression of mRNAs for the de novo enzymes that synthesize FA from precursor molecules (Osborne, 2001; Horton et al., 2003). In vivo deletion of $S C A P$ in the mouse mammary gland significantly decreases FA synthesis and the growth rate of nursing pups (Rudolph et al., 2010). The bovine mammary gland also uses the SCAP-SREBP regulatory system to establish de novo FA synthesis and to integrate dietary regulation of lipogenic gene expression during lactation (Harvatine and Bauman, 2006). Insulin-induced gene 1 is a key regulator in the processing of SREBP1, which mediates its posttranslational maturation and activation ( $\mathrm{Li}$ et al., 2003). Anchoring of SREBP1 in the endoplasmic reticulum (ER) as part of a tripartite complex with SCAP and INSIG1 (Eberlé et al., 2004) exerts a permissive retaining effect on SREBP1, preventing its maturation and activation. If the ER-anchoring activity of INSIG1 is diminished, SCAP escorts SREBP1 from the ER to the Golgi to be cleaved, producing a soluble and transcriptionally active mature SREBP1 protein (Osborne, 2001). In 3T3-L1 adipocytes, overexpression of INSIG1 inhibits both adipogenesis and lipid accumulation ( $\mathrm{Li}$ et al., 2003). In the bovine mammary gland during lactation, the upregulation of INSIG1 can dampen SREBP1 activity (Bionaz and Loor, 2008b). In this study, CIDEC regulated the expression of only $S C A P$ and SREBP1 in mammary epithelial cells of dairy cows. However, INSIG1 expression was not affected by CIDEC.

Nuclear receptor PPAR $\gamma$ is also upregulated in bovine mammary tissue during lactation (Kadegowda et al., 2009). Treatment of bovine mammary epithelial cells with rosiglitazone, a specific PPAR $\gamma$ agonist, results in coordinated upregulation of expression of genes involved in FA import, de novo FA synthesis, and TAG synthesis (Kadegowda et al., 2008). However, we ob- served that expression of $P P A R \gamma$ and its coactivator, PPARG1A, was not affected by CIDEC in cultured mammary epithelial cells of dairy cows, suggesting that $C I D E C$ expression does not feed back on PPAR $\gamma$ expression in dairy cow mammary gland.

\section{CONCLUSIONS}

Cell death-inducing DNA fragmentation factor- $\alpha$-like effector $\mathrm{C}$ is expressed in lactating mammary tissues of dairy cows. It is capable of regulating the expression of genes involved in milk lipid synthesis and modulating mammary epithelial cell TAG levels in lactating mammary tissue of dairy cows.

\section{ACKNOWLEDGMENTS}

The authors declare that no competing interests exist. This work was supported by grants from the National Natural Science Foundation of China (31401109 to Y. L., and 31671285 to X. H.), the University Nursing Program for Young Scholars with Creative Talents in Heilongjiang Province (UNPYSCT-2015010, China), and Young Talents Project of Northeast Agricultural University (14QC03, China).

\section{REFERENCES}

Bauman, D. E., and J. M. Griinari. 2003. Nutritional regulation of milk fat synthesis. Annu. Rev. Nutr. 23:203-227.

Beigneux, A. P., L. Vergnes, X. Qiao, S. Quatela, R. Davis, S. M. Watkins, R. A. Coleman, R. L. Walzem, M. Philips, K. Reue, and S. G. Young. 2006. Agpat6-A novel lipid biosynthetic gene required for triacylglycerol production in mammary epithelium. J. Lipid Res. 47:734-744.

Bionaz, M., and J. J. Loor. 2008a. ACSL1, AGPAT6, FABP3, LPIN1, and SLC27A6 are the most abundant isoforms in bovine mammary tissue and their expression is affected by stage of lactation. J. Nutr. 138:1019-1024.

Bionaz, M., and J. J. Loor. 2008b. Gene networks driving bovine milk fat synthesis during the lactation cycle. BMC Genomics 9:366.

Brown, M. S., and J. L. Goldstein. 1997. The SREBP pathway: Regulation of cholesterol metabolism by proteolysis of a membranebound transcription factor. Cell 89:331-340.

Connor, E. E., S. Siferd, T. H. Elsasser, C. M. Evock-Clover, C. P. Van Tassell, T. S. Sonstegard, V. M. Fernandes, and A. V. Capuco. 2008. Effects of increased milking frequency on gene expression in the bovine mammary gland. BMC Genomics 9:362.

Eberlé, D., B. Hegarty, P. Bossard, P. Ferré, and F. Foufelle. 2004 SREBP transcription factors: Master regulators of lipid homeostasis. Biochimie 86:839-848.

Emery, R. S. 1973. Biosynthesis of milk fat. J. Dairy Sci. 56:1187-1195.

Farese, R. V. Jr., S. Cases, and S. J. Smith. 2000. Triglyceride synthesis: Insights from the cloning of diacylglycerol acyltransferase. Curr. Opin. Lipidol. 11:229-234.

Harvatine, K. J., and D. E. Bauman. 2006. SREBP1 and thyroid hormone responsive spot 14 (S14) are involved in the regulation of bovine mammary lipid synthesis during diet-induced milk fat depression and treatment with CLA. J. Nutr. 136:2468-2474.

Hillerton, J. E., C. H. Knight, A. Turvey, S. D. Wheatley, and C. J. Wilde. 1990. Milk yield and mammary function in dairy cows milked four times daily. J. Dairy Res. 57:285-294. 
Horton, J. D., N. A. Shah, J. A. Warrington, N. N. Anderson, S. W. Park, M. S. Brown, and J. L. Goldstein. 2003. Combined analysis of oligonucleotide microarray data from transgenic and knockout mice identifies direct SREBP target genes. Proc. Natl. Acad. Sci. USA 100:12027-12032.

Hou, X., L. Lin, W. Xing, Y. Yang, X. Duan, Q. Li, X. Gao, and Y. Lin. 2016. Spleen tyrosine kinase regulates mammary epithelial cell proliferation in mammary glands of dairy cows. J. Dairy Sci. 99:3858-3868.

Kadegowda, A. K., M. Bionaz, L. S. Piperova, R. A. Erdman, and J. J. Loor. 2008. Lipogenic gene expression in MAC-T cells is affected differently by fatty acids and enhanced by PPAR-gamma activation. J. Dairy Sci. 91(E-Suppl. 1):566.

Kadegowda, A. K., M. Bionaz, L. S. Piperova, R. A. Erdman, and J. J. Loor. 2009. Peroxisome proliferator-activated receptor-gamma activation and long-chain fatty acids alter lipogenic gene networks in bovine mammary epithelial cells to various extents. J. Dairy Sci. 92:4276-4289.

Kang, Y., S. Hengbo, L. Jun, L. Jun, Z. Wangsheng, T. Huibin, and S. Huaiping. 2015. PPARG modulated lipid accumulation in dairy GMEC via regulation of ADRP gene. J. Cell. Biochem. 116:192201.

Keller, P., J. T. Petrie, P. De Rose, I. Gerin, W. S. Wright, S. H. Chiang, A. R. Nielsen, C. P. Fischer, B. K. Pedersen, and O. A. MacDougald. 2008. Fat-specific protein 27 regulates storage of triacylglycerol. J. Biol. Chem. 283:14355-14365.

Li, J., K. Takaishi, W. Cook, S. K. McCorkle, and R. H. Unger. 2003. Insig-1 "brakes" lipogenesis in adipocytes and inhibits differentiation of preadipocytes. Proc. Natl. Acad. Sci. USA 100:9476-9481.

Ma, L., and B. A. Corl. 2012. Transcriptional regulation of lipid synthesis in bovine mammary epithelial cells by sterol regulatory element binding protein-1. J. Dairy Sci. 95:3743-3755.

Marshall, M. O., and J. Knudsen. 1979. Specificity of diacylglycerol acyltransferase from bovine mammary gland, liver and adipose tissue towards acyl-CoA esters. Eur. J. Biochem. 94:93-98.

Martin, D. B., M. G. Horning, and P. R. Vagelos. 1961. Fatty acid synthesis in adipose tissue. I. Purification and properties of a long chain fatty acid-synthesizing system. J. Biol. Chem. 236:663-668.

Mashek, D. G., and R. A. Coleman. 2006. Cellular fatty acid uptake: The contribution of metabolism. Curr. Opin. Lipidol. 17:274-278.

Matsusue, K., T. Kusakabe, T. Noguchi, S. Takiguchi, T. Suzuki, S. Yamano, and F. J. Gonzalez. 2008. Hepatic steatosis in leptindeficient mice is promoted by the PPARgamma target gene Fsp27. Cell Metab. 7:302-311.

McManaman, J. L. 2009. Formation of milk lipids: A molecular perspective. Clin. Lipidol. 4:391-401.
Mellenberger, R. W., D. E. Bauman, and D. R. Nelson. 1973. Metabolic adaptations during lactogenesis. Fatty acid and lactose synthesis in cow mammary tissue. Biochem. J. 136:741-748.

Nishino, N., Y. Tamori, S. Tateya, T. Kawaguchi, T. Shibakusa, W Mizunoya, K. Inoue, R. Kitazawa, S. Kitazawa, Y. Matsuki, R Hiramatsu, S. Masubuchi, A. Omachi, K. Kimura, M. Saito, T. Amo, S. Ohta, T. Yamaguchi, T. Osumi, J. Cheng, T. Fujimoto, H. Nakao, K. Nakao, A. Aiba, H. Okamura, T. Fushiki, and M. Kasuga. 2008. FSP27 contributes to efficient energy storage in murine white adipocytes by promoting the formation of unilocular lipid droplets. J. Clin. Invest. 118:2808-2821.

Osborne, T. F. 2001. CREating a SCAP-less liver keeps SREBPs pinned in the ER membrane and prevents increased lipid synthesis in response to low cholesterol and high insulin. Genes Dev. 15:1873-1878.

Puri, V., S. Konda, S. Ranjit, M. Aouadi, A. Chawla, M. Chouinard, A. Chakladar, and M. P. Czech. 2007. Fat-specific protein 27, a novel lipid droplet protein that enhances triglyceride storage. J. Biol. Chem. 282:34213-34218.

Rudolph, M. C., J. Monks, V. Burns, M. Phistry, R. Marians, M. R. Foote, D. E. Bauman, S. M. Anderson, and M. C. Neville. 2010. Sterol regulatory element binding protein and dietary lipid regulation of fatty acid synthesis in the mammary epithelium. Am. J. Physiol. Endocrinol. Metab. 299:E918-E927.

Schmittgen, T. D., and K. J. Livak. 2008. Analyzing real-time PCR data by the comparative C(T) method. Nat. Protoc. 3:1101-1108.

Smith, S. 2009. Mechanism of chain length determination in biosynthesis of milk fatty acids. 1980. J. Mammary Gland Biol. Neoplasia 14:245-260.

Smith, S. J., S. Cases, D. R. Jensen, H. C. Chen, E. Sande, B. Tow, D. A. Sanan, J. Raber, R. H. Eckel, and R. V. Jr. Farese. 2000. Obesity resistance and multiple mechanisms of triglyceride synthesis in mice lacking Dgat. Nat. Genet. 25:87-90.

Steinberg, R. S., L. Pereira, G. A. Lacorte, M. G. Peixoto, R. S. Verneque, R. L. Teodoro, M. A. Machado, C. G. Fonseca, and M. R. Carvalho. 2009. Technical note: A new and cost-effective method for detection of the bovine acyl-CoA:diacylglycerol acyltransferase $1 \mathrm{~K} 232 \mathrm{~A}$ polymorphism in cattle. J. Dairy Sci. 92:773-776.

Tan, J. S., C. J. Seow, V. J. Goh, and D. L. Silver. 2014. Recent advances in understanding proteins involved in lipid droplet formation, growth and fusion. J. Genet. Genomics 41:251-259.

Toh, S. Y., J. Gong, G. Du, J. Z. Li, S. Yang, J. Ye, H. Yao, Y. Zhang, B. Xue, Q. Li, H. Yang, Z. Wen, and P. Li. 2008. Up-regulation of mitochondrial activity and acquirement of brown adipose tissuelike property in the white adipose tissue of fsp27 deficient mice. PLoS One 3:e2890. 